\title{
Estudio de la respuesta hidrológica en la cuenca urbana de montaña San Luis-Palogrande
}

\section{Assessment of runoff response in San Luis - Palogrande small urban mountain watershed}

\author{
Diana Rey-Valencia ${ }^{1}$, Jeannette Zambrano ${ }^{2}$ \\ ${ }^{1}$ Grupo de trabajo académico en Ingeniería Hidráulica y Ambiental, Departamento de Ingeniería Civil, Universidad Nacional de \\ Colombia, Colombia, Email: dimreyva@unal.edu.co \\ ${ }^{2}$ Grupo de trabajo académico en Ingeniería Hidráulica y Ambiental, Profesora asociada, Departamento de Ingeniería Civil, \\ Universidad Nacional de Colombia, Colombia, Email: jdzambranona@unal.edu.co
}

RECIBIDO: Abril 10, 2017. ACEPTADO: Junio 13, 2017. VERSIÓN FINAL: Octubre 12, 2017.

\begin{abstract}
RESUMEN
Este trabajo presenta una modelación hidrológica con HEC-HMS de una pequeña cuenca experimental urbana, ubicada en zona montañosa de la ciudad de Manizales, Colombia. El estudio se realiza para conocer la respuesta hidrológica en cuencas urbanas de alta montaña, donde el aumento del proceso de urbanización ha producido modificaciones en la dinámica natural de las cuencas, trayendo como consecuencia aumentos en los volúmenes de escorrentía, en los caudales punta y en las velocidades de flujo, lo que a su vez incrementa el riesgo a inundaciones pluviales. Esta problemática es más crítica en cuencas urbanas de montaña ya que las altas pendientes aumentan aún más las velocidades de flujo. En este tipo de cuencas se tienen pocos estudios. Del análisis realizado en la cuenca seleccionada se encuentra que el uso de suelo ejerce mayor control de las altas pendientes en la respuesta hidrológica.
\end{abstract}

PALABRAS CLAVE: Escorrentía superficial; pequeñas cuencas de montaña; modelación hidrológica.

\begin{abstract}
Here is presented a HEC-HMS hydrological modeling in a small experimental urban watershed, located in a mountainous area of the city of Manizales, Colombia. The study is realized to determine hydrological response in high mountain urban watersheds, where urbanization has created an acute problem relating to floods. This process has produced changes in natural dynamics of watersheds, resulting increases in runoff volumes, peak flows and flow velocities, what in turn increases the floods risk. This problem is most critical in urban mountain watersheds, due to steep slopes, it rises even more flow velocities. From the analysis in the selected watershed, it was found that land use exerts greater control over the hydrological response than high slopes.
\end{abstract}

KEYWORDS: Urban runoff; small mountain urban watersheds; hydrological modelling.

\section{INTRODUCCIÓN}

Durante el siglo XXI el desarrollo de las urbanizaciones se ha acelerado considerablemente debido a la dinámica poblacional actual. En el caso de Colombia en el año
2005 se tiene un porcentaje de población urbana de $74.3 \%$ presentándose un gran aumento con respecto al año 1938 donde el $29.1 \%$ de la población habitaba en los centros urbanos [1]. 
Este proceso de urbanización ha modificado considerablemente el paisaje de las cuencas, ya que la construcción de infraestructuras modifica el uso del suelo. Estas modificaciones han generado el aumento del área impermeabilizada y la disminución o eliminación de la cobertura vegetal. Lo anterior ha traído como consecuencia cambios en la hidrología como la disminución de la infiltración y el consecuente aumento del volumen de escorrentía superficial, el incremento de las velocidades del flujo superficial y dentro de la red, así como el aumento del caudal de escorrentía pico y la disminución del tiempo para alcanzar este (tiempo pico) [2] Figura1. Los mayores volúmenes de escorrentía producen fallos hidráulicos en la red, además de la disminución de la capacidad hidráulica producida por el ingreso de sedimentos. Esto genera inundaciones pluviales cada vez más recurrentes, causando detrimento económico en las ciudades.

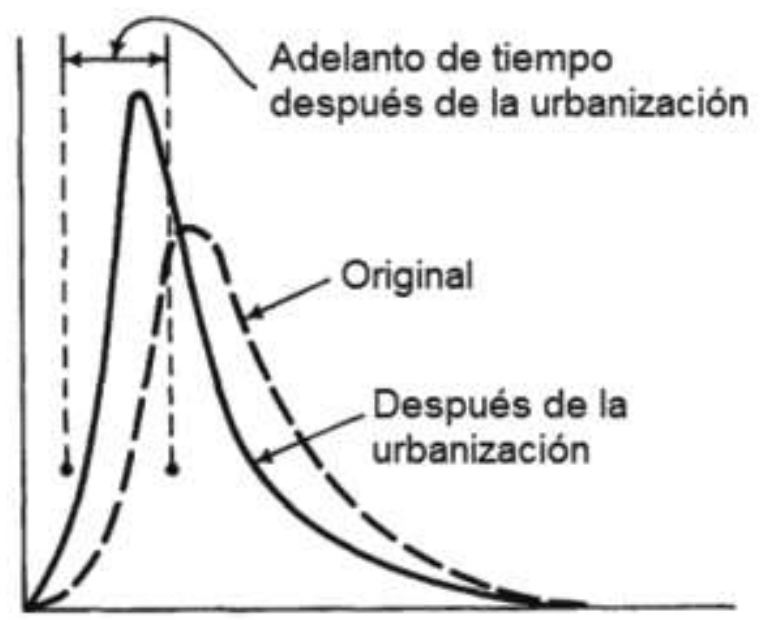

Figura 1. Hidrograma antes y después de la urbanización Fuente: Adaptado de [2].

El fenómeno de las inundaciones pluviales se viene presentado a nivel mundial a medida que se incrementa el nivel de urbanización de las cuencas como ha sido documentado en varios estudios [3][4][5][6][7][8][9], sin embargo, el estudio de esta problemática se ha realizado en su mayoría en cuencas donde los cambios de pendiente no son tan fuertes.

En el caso de cuencas con altas o medias pendientes como aquellas propias de la región andina colombiana, la problemática se agrava ya que el proceso de transformación lluvia-escorrentía es más rápido y por ende se presenta una mayor velocidad superficial y dentro de la red. Por tal razón, es importante estudiar el comportamiento hidrológico e hidráulico de las cuencas urbanas para conocer las modificaciones hidrológicas causadas por los procesos de urbanización y su afectación en la red de alcantarillado.

Por tanto, en esta investigación se analiza la respuesta hidrológica en cuencas urbanas de media y alta montaña con el objetivo de caracterizar su respuesta hidrológica diferenciando entre una zona urbana y una zona poco intervenida. Con este fin, se realiza modelación hidrológica en la Cuenca Experimental Urbana San LuisPalogrande, ubicada en la ciudad de Manizales, con características de alta montaña.

\section{METODOLOGÍA}

\subsection{Modelo utilizado}

Se utilizó un modelo de base física, ya que este permite explicar los procesos que generan flujo desde el ingreso hasta la salida de la cuenca. Se emplean este tipo de modelos, ya que permiten singularizar la marcada variabilidad espacial de los diferentes parámetros que se presentan en las cuencas urbanas [10] [11] [12] [13]. Para tal fin se utilizará el modelo HEC-HMS, modelo de base física semi-distribuido, que conceptualiza la cuenca por medio de unidades hidrológicas homogéneas, y representa los procesos de interceptación, almacenamiento superficial, infiltración, escorrentía, caudal base y tránsito del hidrograma.

El modelo propuesto en HEC-HMS simula la infiltración por medio de Green-Ampt [14], la transformación lluviaescorrentía y el tránsito del hidrograma por medio del modelo unidimensional de Onda Cinemática [15]. Para la cuenca la infiltración ha sido revisada con el método del CN-SCS [16], encontrándose un ajuste poco satisfactorio. Adicionalmente, los sondeos realizados en los suelos de la cuenca, permite el uso de Green-Ampt.

No se tuvo en cuenta la interceptación debido a que representa una parte muy pequeña de las pérdidas totales, y el caudal base fue separado previamente en el hidrograma observado por medio del método gráfico.

El modelo Green y Ampt determina las pérdidas por infiltración, utilizando la ley de Darcy y considerando la propagación del frente húmedo (límite entre el suelo saturado y no saturad o) como un flujo en pistón [17] obteniendo la ecuación (1) y ecuación (2) para la infiltrabilidad y la infiltración acumulada respectivamente [15].

$$
\begin{gathered}
\mathrm{f}_{\mathrm{c}}(\mathrm{t})=K_{s}\left(\frac{\psi(\eta-\theta i)}{\mathrm{I}(\mathrm{t})}+1\right) \\
\mathrm{I}(\mathrm{t})=K_{s} \mathrm{t}+\psi(\eta-\theta i) * \ln \left(1+\frac{\mathrm{I}(\mathrm{t})}{\psi(\eta-\theta i)}\right)
\end{gathered}
$$


$\mathbf{f}_{\mathbf{c}}=$ Tasa de infiltración $[\mathrm{mm}]$

$\mathbf{I}(\mathbf{t})=$ Infiltración acumulada [mm]

$\mathbf{K}_{\mathbf{s}}=$ Conductividad Hidráulica $[\mathrm{mm} / \mathrm{h}]$

$\boldsymbol{\eta}=$ Porosidad [adim]

$\boldsymbol{\theta}_{\mathbf{i}}=$ Humedad inicial [adim]

$\boldsymbol{\psi}=$ Potencial de succión del frente húmedo [mm]

El modelo de Onda Cinemática describe el flujo en planos que entregan a un canal, de manera que se calcula el caudal por unidad de ancho utilizando la precipitación neta la que se calcula como la diferencia entre las tasas de precipitación e infiltración [11]. La formulación matemática de este modelo no tiene solución analítica, de manera que se resuelve por medio de métodos numéricos. Este modelo permite simular el hidrograma a la salida para un evento de lluvia acumulando el flujo de los planos localizados en la cuenca. Su ventaja es que soluciona ecuaciones físicas que rigen el flujo superficial, siendo la solución para flujo unidimensional la descrita en la ecuación (3) [15].

$$
\frac{\partial \mathrm{h}}{\partial \mathrm{t}}+\frac{\partial \mathrm{q}}{\partial \mathrm{x}}=\mathrm{P}_{\mathrm{N}}(\mathrm{x}, \mathrm{t})
$$

$\mathbf{P n}=$ Precipitación neta $[\mathrm{mm}]$

$\mathbf{q}=$ Caudal por unidad de ancho $\left[\mathrm{m}^{2} / \mathrm{s}\right]$

$\mathbf{t}=$ Tiempo [s]

$\mathbf{x}=$ Desplazamiento $[\mathrm{m}]$

$\mathbf{h}=$ Calado del agua $[\mathrm{m}]$

\subsection{Zona de estudio}

La Cuenca Experimental Urbana San Luis-Palogrande se encuentra ubicada en la ciudad de Manizales, Caldas, Colombia la que a su vez se encuentra en la región andina sobre la cordillera de los Andes (cordillera central). La cuenca San Luis-Palogrande es afluente del río Chinchiná encontrándose en la vertiente norte de éste, con un área total de aproximadamente $2.5 \mathrm{~km}^{2}$ [18] [19] Tabla 1. El suelo en la cuenca es destinado principalmente a uso residencial, incluyendo parte de la zona educativa de la ciudad que corresponde a un $69 \%$, así como una amplia zona de bosques que corresponde a un $31 \%$ para el año 2005. La cuenca tiene una topografía abrupta con un rango altitudinal de 2166 a 1974 m.s.n.m, y sus principales características morfométricas se describen en la Tabla 1.

La cuenca está instrumentada desde el año 2006, con 2 estaciones pluviométricas, 1 meteorológica y 1 estación hidrometeorológica (estación pluviométrica e hidrométrica) [20]. La instrumentación no se encuentra en toda la cuenca, por tanto del área total se seleccionó una zona para la modelación a la que se denomina San Luis Ruta 30, con un área aproximada de $1 \mathrm{~km}^{2}$. En esta zona el régimen de precipitación sigue un comportamiento bimodal presentándose valores máximos en los meses de Abril (204.5 mm) y Octubre (261.3 mm), y varía espacialmente de manera considerable. En la cuenca la precipitación promedio anual es de $1903.6 \mathrm{~mm}$, la máxima anual de $2720.5 \mathrm{~mm}$ y la mínima anual de $1378.1 \mathrm{~mm}$.

Tabla 1. Características morfométricas.

\begin{tabular}{|c|c|c|c|}
\hline Parámetro & $\begin{array}{c}\text { W40 } \\
\text { Zona } \\
\text { alta }\end{array}$ & $\begin{array}{c}\text { W50 } \\
\text { Zona } \\
\text { alta }\end{array}$ & $\begin{array}{c}\text { W60 } \\
\text { Zona } \\
\text { baja }\end{array}$ \\
\hline Área (A) $\left[\mathrm{km}^{2}\right]$ & 0.45 & 0.17 & 0.26 \\
\hline Perímetro (P) [km] & 3.78 & 2.21 & 3.72 \\
\hline Longitud (L) [km] & 0.97 & 0.55 & 0.63 \\
\hline $\begin{array}{c}\text { Longitud máxima (Lm) } \\
{[\mathrm{km}]}\end{array}$ & 1.23 & 0.84 & 1.28 \\
\hline Pendiente (S) [\%] & 16.11 & 21.19 & 42.31 \\
\hline Ancho (B) [km] & 0.46 & 0.31 & 0.41 \\
\hline Factor de forma (Kf) & 0.48 & 0.56 & 0.66 \\
\hline $\begin{array}{c}\text { Coeficiente de } \\
\text { compacidad (Kc) }\end{array}$ & 1.58 & 1.5 & 2.04 \\
\hline Desnivel altitudinal (h) & 89.74 & 108.29 & 152.00 \\
\hline $\begin{array}{c}\text { Longitud total de } \\
\text { drenajes (Ld) }[\mathrm{km}]\end{array}$ & 0.19 & $\mathrm{n} / \mathrm{a}$ & 0.73 \\
\hline $\begin{array}{c}\text { Densidad de drenaje } \\
\left.\text { (Dd) [km/km }{ }^{2}\right]\end{array}$ & 0.42 & $\mathrm{n} / \mathrm{a}$ & 2.81 \\
\hline$\%$ área impermeable & 52 & 52 & 14 \\
\hline
\end{tabular}

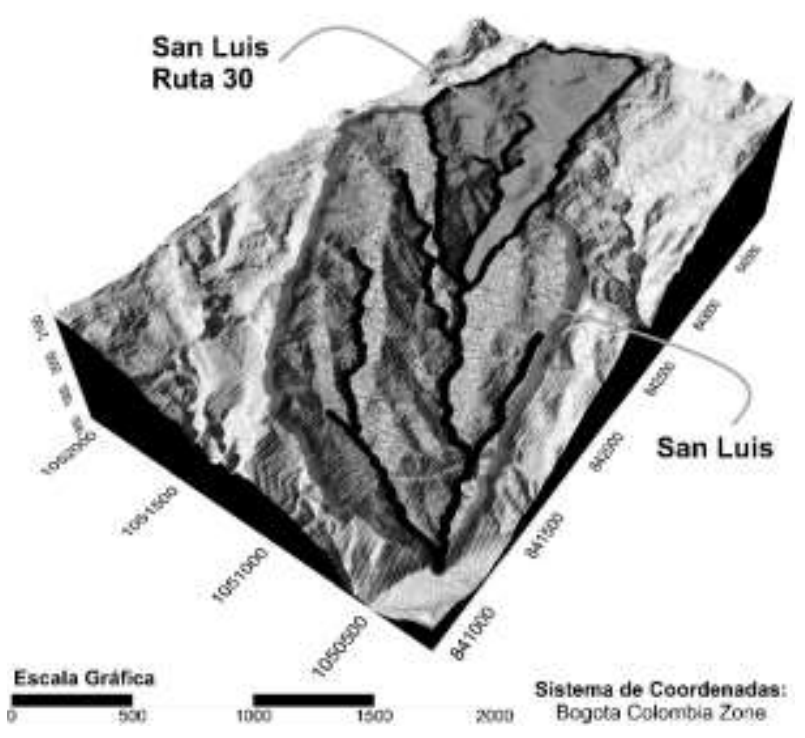

Figura 2. Vista 3D de la cuenca San Luis Ruta 30 Fuente: Elaboración propia.

La selección de las subcuencas se realizó diferenciando la zona urbana (zona alta) de la zona menos intervenida (zona baja), utilizando la herramienta HEC-GeoHMS. La zona alta tiene un alto nivel de impermeabilidad, con pendientes medias y bajas y una pendiente promedio de 
$18 \%$, y alta densidad del alcantarillado (subcuencas W40 y W50). En la parte baja se presentan pendientes altas (especialmente cerca del cauce) y una pendiente promedio del $42.3 \%$ y poca red de alcantarillado (denominada subcuenca W60).

Para la modelación se contó con el Modelo de Elevación Digital MED de resolución de 2×2 m, el mapa de usos de suelo fue construido mediante fotografía aérea y el mapa de suelos generado a partir de la Microzonificación Sísmica de la ciudad de Manizales [21] (Figura 3).

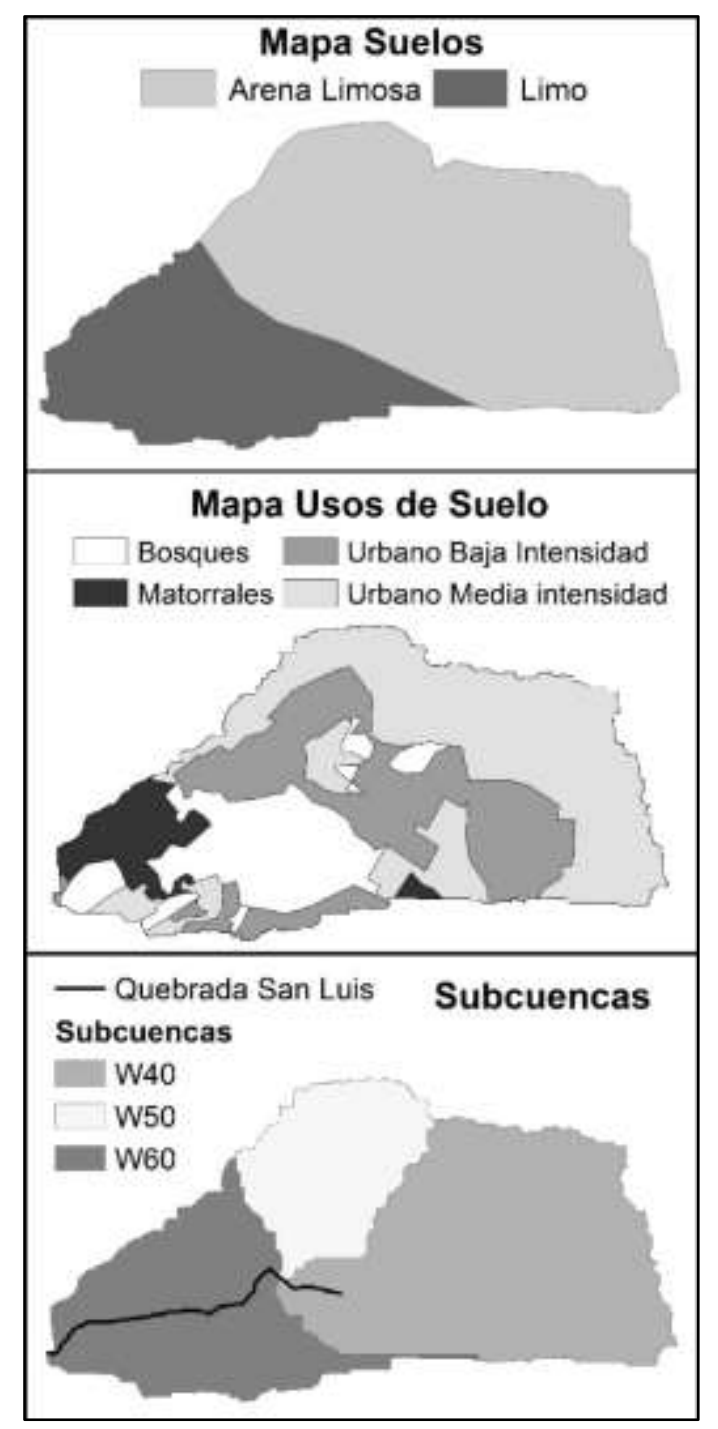

Figura 3. Mapa de las características de la cuenca San Luis Ruta 30 Fuente: Elaboración propia.

La conceptualización de la subcuenca se realizó por medio de 2 planos en cada subcuenca con la ayuda de ArcGis, tomando en la zona urbana (W40 y W50) la división como el camino más largo de la red de alcantarillado y en la zona menos intervenida (W60) el cauce. Para el tránsito del hidrograma los parámetros corresponden la quebrada San Luis en la parte baja.

\subsection{Calibración y validación}

A partir de la información de precipitación, se extrajeron 9 eventos para calibración y 15 más para validación (Tabla 2), tomando precipitaciones mayores a $10 \mathrm{~mm}$ los que se ponderaron por medio de polígonos de Thiessen.

Tabla 2. Eventos de calibración y validación.

\begin{tabular}{|c|c|c|c|}
\hline \multicolumn{4}{|c|}{ Calibración } \\
\hline Fecha & \# & $\begin{array}{l}\text { Precipitación total } \\
{[\mathrm{mm}]}\end{array}$ & $\begin{array}{c}\text { Duración } \\
\text { [min] }\end{array}$ \\
\hline $28 / 11 / 2009$ & 1 & 20.0 & 80 \\
\hline 21/06/2009 & 2 & 18.1 & 135 \\
\hline $01 / 07 / 2010$ & 3 & 16.8 & 50 \\
\hline 14/04/2011 & 4 & 15.6 & 55 \\
\hline $19-20 / 04 / 2011$ & 5 & 18.0 & 130 \\
\hline $18 / 06 / 2009$ & 6 & 11.9 & 65 \\
\hline $26 / 10 / 2009$ & 7 & 15.0 & 50 \\
\hline $07 / 04 / 2009$ & 8 & 13.6 & 85 \\
\hline $18 / 10 / 2009$ & 9 & 11.1 & 65 \\
\hline \multicolumn{4}{|c|}{ Validación } \\
\hline Fecha & \# & $\begin{array}{c}\text { Precipitación } \\
\text { total }[\mathrm{mm}]\end{array}$ & $\begin{array}{c}\text { Duración } \\
\text { [min] }\end{array}$ \\
\hline $16 / 03 / 2009$ & 1 & 10.6 & 65 \\
\hline $02 / 10 / 2009$ & 2 & 11.1 & 75 \\
\hline $05-06 / 04 / 2010$ & 3 & 17.3 & 65 \\
\hline $17 / 02 / 2011$ & 4 & 10.6 & 115 \\
\hline $10 / 11 / 2009$ & 5 & 18.2 & 95 \\
\hline $26 / 01 / 2010$ & 6 & 18.7 & 125 \\
\hline $10 / 03 / 2010$ & 7 & 17.8 & 65 \\
\hline $02 / 03 / 2011$ & 8 & 18.2 & 110 \\
\hline $09 / 03 / 2011$ & 9 & 17.5 & 110 \\
\hline $06-07 / 10 / 2012$ & 10 & 22.4 & 155 \\
\hline $01 / 03 / 2011$ & 11 & 29.5 & 100 \\
\hline $02 / 03 / 2011$ & 12 & 29.0 & 95 \\
\hline $25 / 02 / 2011$ & 13 & 29.2 & 65 \\
\hline $24-25 / 01 / 2009$ & 14 & 24.8 & 130 \\
\hline 03/03/2011 & 15 & 47.8 & 135 \\
\hline
\end{tabular}

Para la determinación de las propiedades hidrológicas asociadas a los suelos se utilizaron las recomendaciones de [22], teniendo en cuenta que en la zona se reporta Arena Limosa en la parte alta y Limos en la parte baja [19] (Tabla 3). Por otra parte, los parámetros hidráulicos asociados al uso y cobertura del suelo fueron obtenidos siguiendo las recomendaciones del Departamento de Agricultura de los Estados Unidos (Multi Resolution Land Characteristics) [23] (Tabla 4). 
Tabla 3. Parámetros hidrológicos iniciales según el suelo.

\begin{tabular}{|c|c|c|c|c|c|}
\hline Sub-cuenca & Suelo & $\eta[$ adim $]$ & $\begin{array}{c}\mathrm{K} \\
{[\mathrm{mm} / \mathrm{h}]}\end{array}$ & $(\Psi)[\mathrm{mm}]$ & $\theta_{\mathrm{i}}[\mathrm{adim}]$ \\
\hline W40 & Arenas limosas & \multirow{2}{*}{0.5} & 20 & 350 & 0.1 \\
\hline W50 & Limos & & 4 & 100 & \\
\hline W60 & & & 20 & \\
\hline
\end{tabular}

Tabla 4. Parámetros hidráulicos iniciales según el uso del suelo.

\begin{tabular}{|c|c|c|}
\hline Uso del suelo & Descripción & $\begin{array}{c}\text { Coeficiente de rugosidad } \\
\text { de Manning (n) }\end{array}$ \\
\hline Bosques & $\begin{array}{c}\text { Áreas caracterizadas por cobertura arbórea (vegetación boscosa natural o semi- } \\
\text { natural, generalmente de alturas mayores a 6 m) }\end{array}$ & 0.600 \\
\hline Matorrales & Áreas dominadas por matorrales. La cobertura será del 25 - 100\% del total & 0.055 \\
\hline $\begin{array}{c}\text { Urbano Intensidad } \\
\text { Baja }\end{array}$ & $\begin{array}{c}\text { Áreas con mezcla entre urbanización y vegetación, mayoritariamente esta } \\
\text { última. Se tendrá áreas impermeable del 20\%. }\end{array}$ & 0.120 \\
\hline $\begin{array}{c}\text { Urbano Intensidad } \\
\text { Media }\end{array}$ & $\begin{array}{c}\text { Áreas con mezcla entre urbanización y vegetación. La superficie impermeable } \\
\text { será de alrededor del 50 -79\% del total de la cobertura. }\end{array}$ & 0.150 \\
\hline
\end{tabular}

Se realizó calibración manual, con el fin de que los valores finales tengan sentido físico, para lo cual partiendo del análisis de sensibilidad del modelo se tomaron como variables de calibración la conductividad hidráulica y el coeficiente de rugosidad de Manning de las subcuencas. Los parámetros calibrados se muestran en las Tabla 5 y Tabla 6.

Tabla 5. Parámetros hidráulicos calibrados según el uso del suelo.

\begin{tabular}{|c|c|}
\hline Uso del suelo & $\begin{array}{c}\text { Coeficiente de rugosidad de } \\
\text { Manning (n) }\end{array}$ \\
\hline Bosques & 0.300 \\
\hline Matorrales & 0.055 \\
\hline Urbano Intensidad Baja & 0.040 \\
\hline Urbano Intensidad Media & 0.012 \\
\hline
\end{tabular}

Tabla 6. Parámetros hidrológicos calibrados según el suelo.

\begin{tabular}{|c|c|c|c|c|c|}
\hline $\begin{array}{c}\text { Sub- } \\
\text { cuenca }\end{array}$ & Suelo & $\begin{array}{c}\eta \\
\text { [adim] }\end{array}$ & $\begin{array}{c}\mathrm{K} \\
{[\mathrm{mm} / \mathrm{h}]}\end{array}$ & $\begin{array}{c}(\Psi) \\
{[\mathrm{mm}]}\end{array}$ & $\begin{array}{c}\theta_{\mathrm{i}} \\
\text { [adim] }\end{array}$ \\
\hline W40 & Arenas & \multirow{3}{*}{0.50} & & 3 & \multirow{3}{*}{0.48} \\
\hline W50 & limosas & & 50 & 350 & \\
\hline W60 & Limos & & 4 & 100 & \\
\hline
\end{tabular}

\section{RESULTADOS Y DISCUSIÓN}

En el proceso de calibración se obtuvieron buenos niveles de ajuste, con coeficientes de Nash superiores a 0.7, errores volumétricos hasta del $10 \%$, como se observa en la Tabla 7. El modelo hidrológico propuesto se enfoca en eventos de respuesta rápida de una precipitación total considerable, dado que este modelo es base para un modelo hidráulico para evaluar el alcantarillado. Con el modelo se logró reproducir adecuadamente el tiempo para llegar al caudal pico para los eventos de lluvia objetivo, de manera que el modelo propuesto reproduce de manera adecuada las respuestas rápidas propias de este tipo de cuencas como se observa en la Figura 4. Sin embargo, para los eventos dobles (con 2 o más caudales pico), eventos de respuesta lenta y eventos menores a los $15 \mathrm{~mm}$ se obtuvieron un nivel de ajuste menor.

Inicialmente, se realizó un análisis de sensibilidad, para determinar la variación de la respuesta ante las modificaciones en los parámetros y seleccionar los parámetros de calibración. Del análisis de sensibilidad se concluye que en la zona menos intervenida la respuesta hidrológica es altamente sensible a los parámetros asociados al suelo especialmente la conductividad hidráulica como se muestra Figura 5(a), presentando variaciones en las simulaciones entre $10 \%$ y $20 \%$ del caudal pico y del volumen total. Por otro lado, en la zona urbana se presenta un comportamiento contrario ya que como se observa en la Figura 5(b) solo se presenta variación cuando la conductividad hidráulica es menor a $20 \mathrm{~mm} / \mathrm{h}$ (simulaciones \#5 y \#6), valor mínimo recomendado por la literatura para este parámetro.

En lo relativo al coeficiente de rugosidad de Manning también cambia según la zona. En la zona urbana se presenta una alta sensibilidad a este parámetro, con aumentos entre simulaciones del caudal pico entre 0.1 a $0.3 \mathrm{~m} 3 / \mathrm{s}$, sin embargo se mantiene constante el volumen total (Figura 6 (b)). Por otro lado, en la zona menos intervenida el modelo no presenta sensibilidad a este parámetro como se observa en la Figura 6(a), teniendo un volumen constante en las diferentes simulaciones y un cambio de $0.1 \mathrm{~m}^{3} / \mathrm{s}$ en el caudal pico.

Una vez calibrados los parámetros, se concluye que el modelo hidrológico propuesto permite estudiar la respuesta hidrológica a diferentes eventos distinguiendo entre la zona urbana y la zona menos intervenida. 
Tabla 7. Resultados del modelo para todos los eventos.

\begin{tabular}{|c|c|c|c|c|c|c|c|c|c|}
\hline \multirow[t]{2}{*}{ Tipo } & \multirow[t]{2}{*}{ \# } & \multicolumn{2}{|c|}{$\begin{array}{c}\text { Volumen total } \\
{\left[1000 \mathrm{~m}^{3}\right]}\end{array}$} & \multirow[t]{2}{*}{$\begin{array}{l}\text { Nash- } \\
\text { Sutcliffe }\end{array}$} & \multicolumn{2}{|c|}{$\begin{array}{c}\text { Caudal punta } \\
{\left[\mathrm{m}^{3} / \mathrm{s}\right]}\end{array}$} & \multicolumn{2}{|c|}{$\begin{array}{l}\text { Tiempo pico } \\
\text { [min] }\end{array}$} & \multirow{2}{*}{$\begin{array}{l}\text { \% error } \\
\text { de } \\
\text { volumen }\end{array}$} \\
\hline & & Observado & Simulado & & Observado & Simulado & Observado & Simulado & \\
\hline \multirow{9}{*}{ 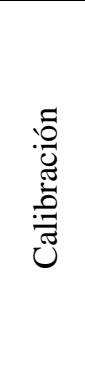 } & 1 & 10.2 & 10.3 & 0.46 & 7 & 6.7 & $15: 15$ & $15: 20$ & -1.0 \\
\hline & 2 & 9.4 & 10.4 & 0.87 & 7.3 & 5.1 & 19:10 & 19:10 & -9.6 \\
\hline & 3 & 8.6 & 6.6 & 0.82 & 6.3 & 5.9 & $17: 10$ & $17: 10$ & 30.3 \\
\hline & 4 & 8.1 & 7.1 & 0.30 & 6.9 & 7 & $13: 45$ & $13: 35$ & 14.1 \\
\hline & 5 & 8.2 & 8.2 & 0.89 & 5.1 & 3.6 & $0: 25$ & $0: 25$ & 0.0 \\
\hline & 6 & 5.6 & 4.3 & 0.91 & 4.1 & 3.2 & $13: 50$ & $13: 50$ & 30.2 \\
\hline & 7 & 7.4 & 5.2 & 0.88 & 6.1 & 5.4 & $14: 40$ & $14: 40$ & 42.3 \\
\hline & 8 & 6.5 & 5.4 & 0.84 & 6.6 & 4.4 & $17: 55$ & $17: 55$ & 20.4 \\
\hline & 9 & 5.6 & 4.6 & 0.79 & 5.6 & 3.1 & $15: 30$ & $15: 30$ & 21.7 \\
\hline \multirow{14}{*}{ 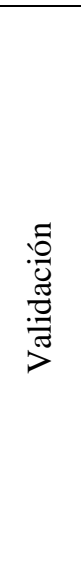 } & 1 & 4.7 & 2.5 & -0.07 & 1.6 & 2.0 & $16: 05$ & $16: 20$ & 88.0 \\
\hline & 2 & 5.1 & 2.1 & -0.49 & 1.8 & 2.3 & $17: 30$ & $17: 30$ & 142.9 \\
\hline & 3 & 8.4 & 7.6 & 0.79 & 3.7 & 3.7 & $23: 35$ & $23: 35$ & 10.5 \\
\hline & 4 & 4.2 & 3.6 & 0.85 & 1.5 & 1.1 & $10: 20$ & $10: 15$ & 16.7 \\
\hline & 5 & 8.9 & 8.1 & 0.89 & 4.9 & 4.7 & $15: 50$ & $15: 40$ & 9.9 \\
\hline & 6 & 9.0 & 7.5 & 0.90 & 5.5 & 4.8 & $14: 35$ & $14: 40$ & 20.0 \\
\hline & 7 & 8.8 & 5.0 & 0.27 & 4.2 & 4.0 & $15: 05$ & $15: 05$ & 76.0 \\
\hline & 8 & 8.5 & 10.4 & 0.73 & 6.5 & 3.9 & $19: 20$ & $19: 15$ & -18.3 \\
\hline & 9 & 8.1 & 8.1 & 0.62 & 2.7 & 2.2 & $13: 50$ & $13: 55$ & 0.0 \\
\hline & 10 & 10.8 & 10.9 & 0.57 & 4.7 & 7.1 & $23: 40$ & $23: 35$ & -0.9 \\
\hline & 11 & 15.4 & 13.4 & 0.75 & 5.7 & 7.3 & $16: 40$ & $16: 30$ & 14.9 \\
\hline & 12 & 14.8 & 14.2 & 0.86 & 6.2 & 5.8 & $14: 25$ & $14: 20$ & 4.2 \\
\hline & 13 & 16.8 & 17.1 & 0.87 & 9.4 & 9.1 & $16: 55$ & $16: 50$ & -1.8 \\
\hline & 14 & 12.1 & 12.6 & 0.85 & 5.1 & 4.2 & $0: 30$ & $0: 30$ & -4.0 \\
\hline
\end{tabular}
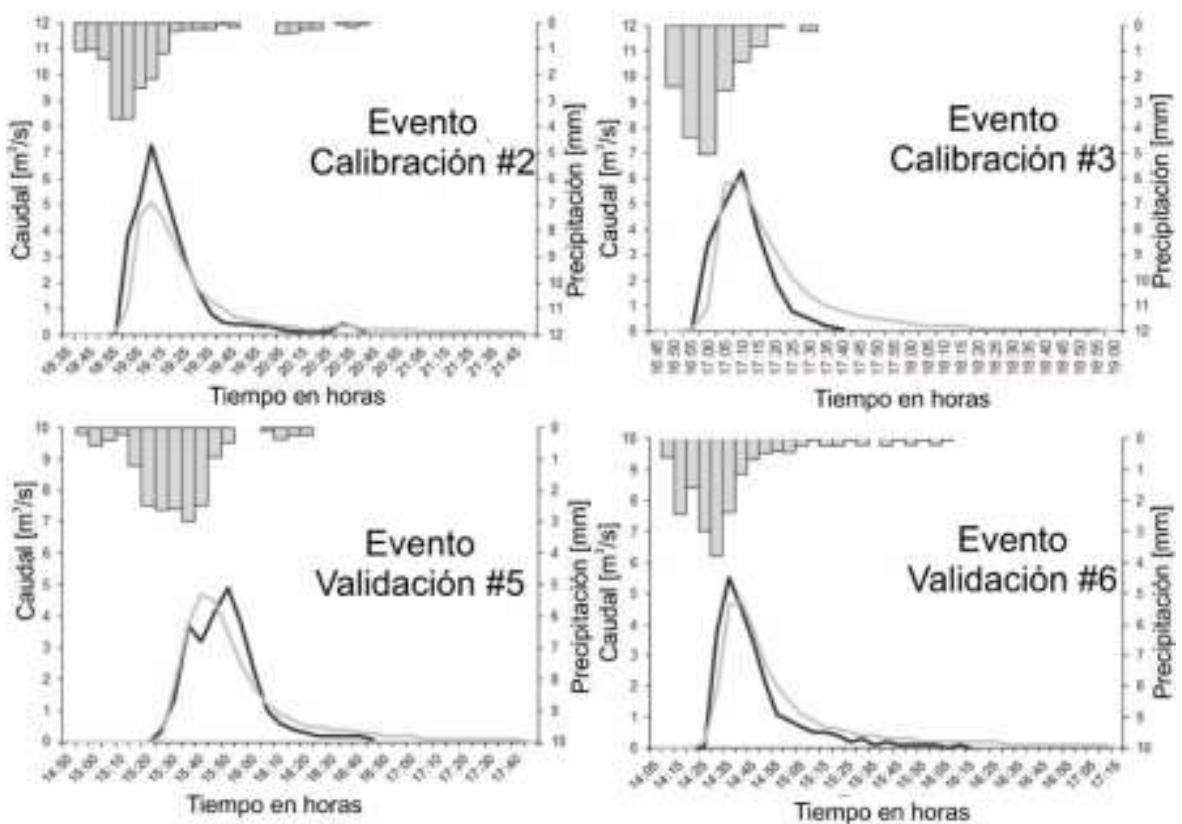

$\square$ Precipitación $[\mathrm{mm}]$ —Caudal Observado [m3/seg] —Caudal Simulado [m3/seg]

Figura 4. Hidrograma observado y simulado ante diferentes eventos de precipitación Fuente: Elaboración propia. 

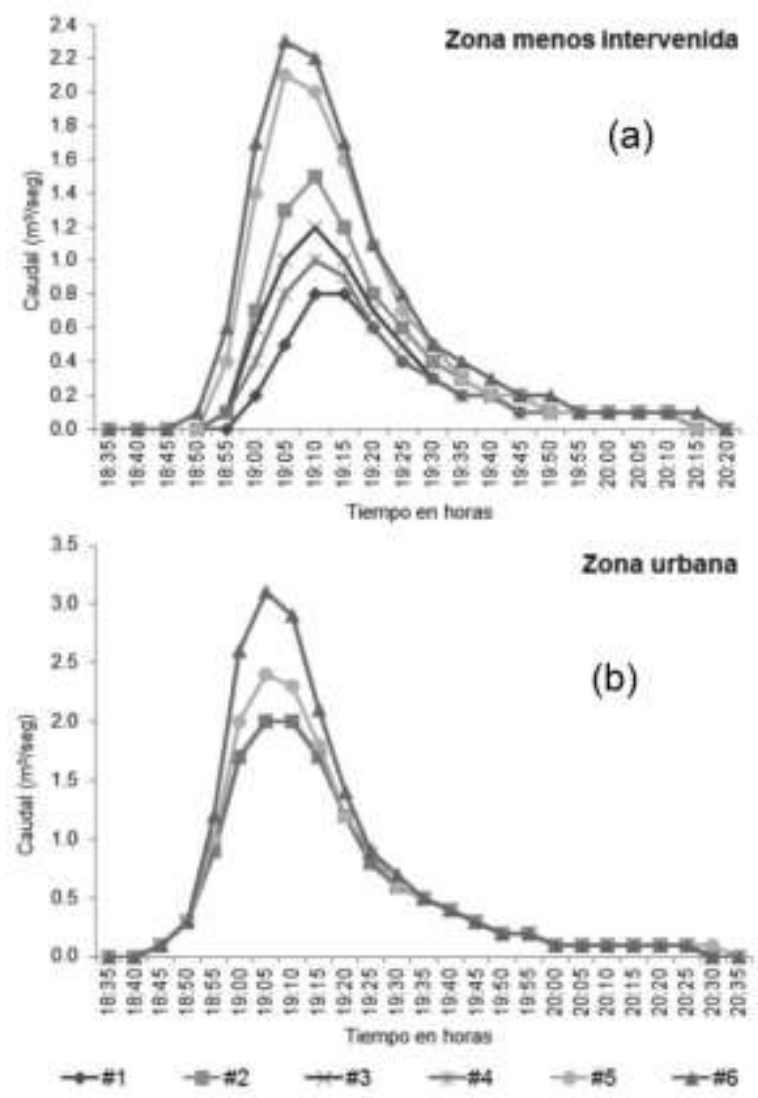

Figura 5. Análisis de sensibilidad para la conductividad hidráulica (a): zona menos intervenida (b): zona urbana Fuente: Elaboración propia.

En la zona urbana se presenta un gran aporte de volumen de escorrentía correspondiendo en promedio al $63.3 \%$ del volumen total, es decir un $1.2 \mathrm{~m}^{3} / \mathrm{m}^{2}$ de volumen unitario (Esto se puede observar en la Figura 7 donde se muestran algunos de los eventos simulados). Mientras que en la zona menos intervenida se presenta una respuesta más atenuada con un menor aporte de volumen (se produce sólo el $36.6 \%$ del volumen total es decir un aporte de 0.9 $\left.\mathrm{m}^{3} / \mathrm{m}^{2}\right)$.

Para todos los eventos simulados en la zona urbana la respuesta hidrológica inicia entre 5 a 10 minutos más rápido que en la zona menos intervenida (Figura 7). Esto se atribuye a las distancias de recorrido que son más cortas y al alto nivel de impermeabilización de esta zona que provocan que la velocidad superficial aumente, por ende, se tiene un proceso transformación lluviaescorrentía más rápido. Todo lo contrario, sucede en la zona menos intervenida donde la cobertura vegetal presente disminuye la velocidad superficial y genera una respuesta hidrológica más atenuada.
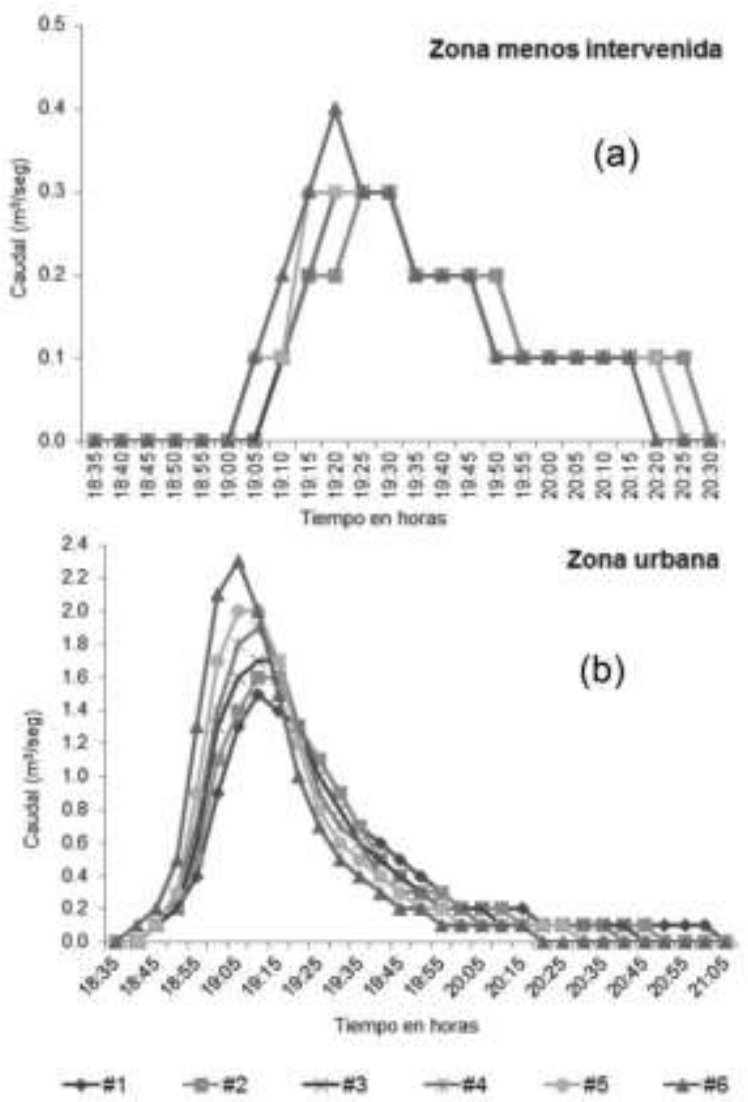

Figura 6. Análisis de sensibilidad para el coeficiente de rugosidad de Manning (a): zona menos intervenida (b): zona urbana Fuente: Elaboración propia.

Así como los mayores volúmenes de escorrentía son generados en la zona urbana (Figura 7). En promedio para los eventos simulados el caudal pico en la zona urbana es de $3.7 \mathrm{~m} 3 / \mathrm{s}$ contra $2.4 \mathrm{~m} 3 / \mathrm{s}$ de la zona menos intervenida, esto es debido al uso de suelo, teniendo en la zona urbana alto porcentaje de impermeabilidad, generando menor volumen de infiltración y por tanto mayores caudales punta.

El tiempo en alcanzar el caudal pico es menor para la zona urbana con respecto a la zona menos intervenida, siendo la diferencia de tiempo entre la cuenca alta y la baja para todos los eventos simulados de alrededor de 5 min. Este comportamiento también se atribuye al uso del suelo que controla la rapidez de la respuesta. Sin embargo, si se revisa el tiempo en alcanzar el caudal pico a la salida de la cuenca es similar al de la zona menos intervenida (Figura 8), debido a que la zona menos intervenida de la cuenca atenúa la respuesta hidrológica retrasándola en promedio $5 \mathrm{~min}$. Si bien es una diferencia pequeña, considerando que es una cuenca menor a un 1 $\mathrm{km}^{2}$, esta diferencia es representativa porque el tiempo de concentración de la cuenca se estima en $22 \min$ [24]. 

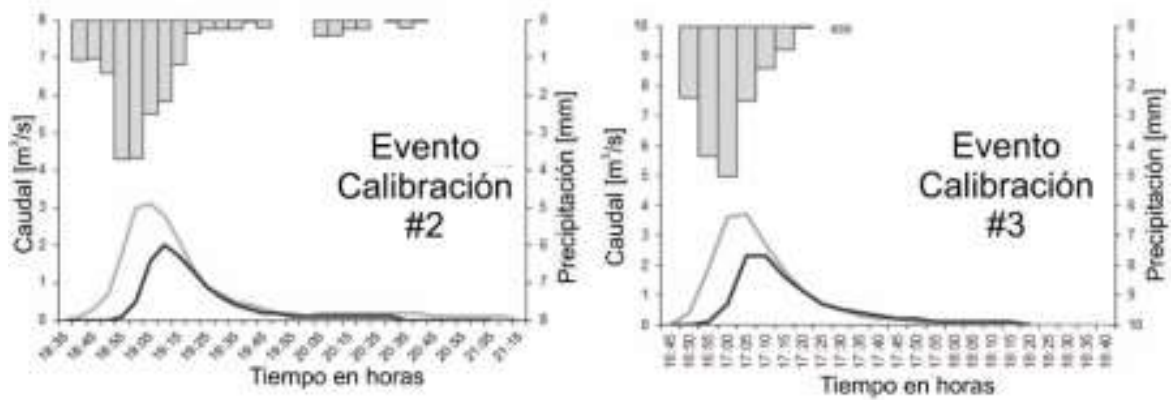

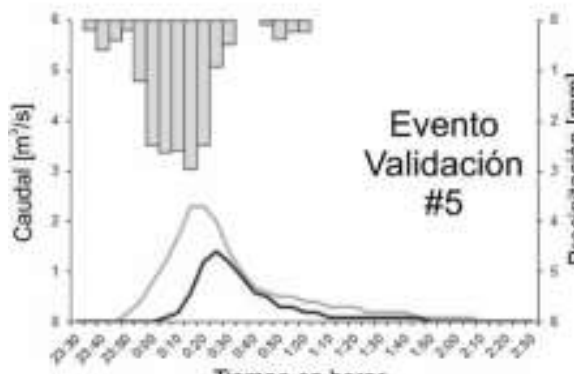

Tiempo en horas

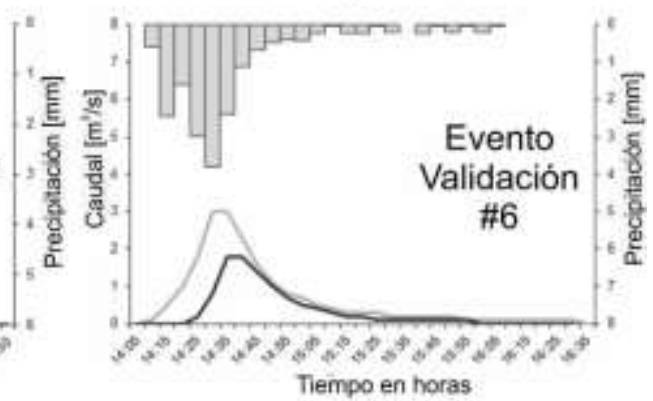

$\square$ Precipitación

- Zona Urbana

- Zona menos intervenida

Figura 7. : Comparación respuesta hidrológica de la zona urbana y la zona menos intervenida ante diferentes eventos de precipitación Fuente: Elaboración propia.
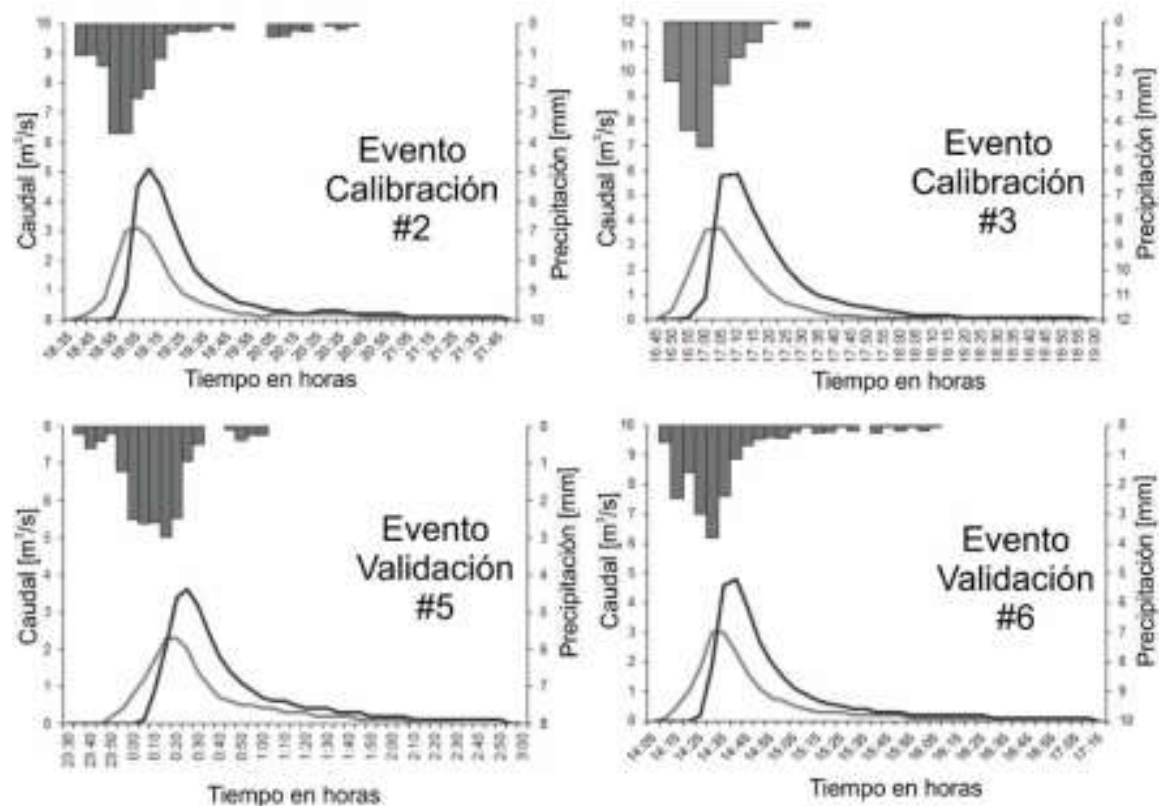

$\rightleftarrows$ Precipitación

-Zona Urbana

- Salida de la cuenca

Figura 8. Comparación respuesta hidrológica de la zona urbana y la salida de la cuenca ante diferentes eventos de precipitación Fuente: Elaboración propia. 
Lo anterior permite concluir que el comportamiento hidrológico cambia entre la zona urbana y la zona menos intervenida, de manera que, en la zona urbana, la respuesta hidrológica está dominada por el uso del suelo, razón por lo cual la respuesta es más rápida y con mayores volúmenes de escorrentía. Todo lo contrario, sucede en la zona menos intervenida de la cuenca, donde la respuesta está dominada por las características hidrológicas del suelo, produciendo mayor infiltración y por tanto un menor volumen de escorrentía.

Por último, se observa que en general para todos los eventos simulados el tiempo de finalización de los hidrogramas simulados difiere del observado, siendo esta diferencia hasta de 1 hora (Figura 4), lo cual se atribuye al nivel de discretización usado en la modelación. Al no lograr ajustar adecuadamente los tiempos, el volumen de escorrentía se reparte en un mayor tiempo de duración y por tanto los caudales punta son subestimados ligeramente.

\section{CONCLUSIONES}

En el presente artículo se da una revisión de la respuesta hidrológica de una cuenca semi-urbanizada con diferencias entre la zona urbana y zona menos intervenida. Se aplicó el modelo hidrológico HEC-HMS a la cuenca urbana San Luis para observar la respuesta hidrológica ante diferentes eventos, analizando la influencia del uso del suelo en dicha respuesta. Para esto se seleccionaron 25 eventos de calibración y validación, para los cuales se obtuvieron niveles de ajuste satisfactorios.

De las simulaciones se concluye que el comportamiento hidrológico cambia entre la zona urbana y la zona menos intervenida, de manera que en la zona urbana la respuesta hidrológica es muy rápida ya que el uso urbano (área muy impermeable) es dominante con respecto a los controles hidrológicos que pueden generar el suelo y otras condiciones naturales. El comportamiento contrario se presenta en la zona menos intervenida con una respuesta más atenuada determinada por las características hidrológicas del suelo las que permiten regular la respuesta hidrológica.

Se comprobó que el uso y cobertura de suelo dominantemente urbano transforma la respuesta hidrológica, ya que el porcentaje de área impermeable controla los volúmenes de escorrentía que se generan. Por otra parte, el coeficiente de rugosidad de Manning controla la rapidez de la respuesta hidrológica, ya que al disminuirlo la duración del hidrograma de toda la cuenca también se disminuye y generalmente se incrementa el caudal pico.
Igualmente, se encontró que el tiempo para alcanzar el caudal pico es mayor para la zona menos intervenida con respecto a la zona urbana. Sin embargo, la respuesta total la cuenca de estudio no es tan rápida dado que es atenuada en la zona menos intervenida por el uso de suelo muy poco urbano, con zonas más vegetalizadas.

El modelo hidrológico propuesto funciona de manera correcta para eventos de respuesta rápida de una precipitación total considerable, sin embargo en el caso de eventos dobles, de respuesta lenta y menores a los 15 $\mathrm{mm}$ es necesario mejorar el nivel de discretización del modelo, para lo cual se deben aplicar modelos con más unidades hidrológicas o distribuidos con una resolución adecuada.

Las cuencas de montaña se caracterizan por su respuesta rápida producida por las altas pendientes. En el modelo propuesto se encontró que la respuesta hidrológica para la cuenca de estudio es rápida, con algunas modificaciones según la zona a estudiar. Para la zona urbana con una pendiente promedio del $18 \%$ y área impermeable del $52 \%$ la respuesta es muy rápida, mientras que en la zona menos intervenida con una pendiente promedio mayor al $43.5 \%$ y un área impermeable menor del $9 \%$, se da una respuesta mucho menos rápida. Por lo que se concluye que aunque la topografía determina parcialmente la respuesta hidrológica, lo más determinante en este caso es el uso del suelo.

El estudio de la respuesta hidrológica de cuencas urbanas es de relevancia dado que la hidrología determina el comportamiento de una red de alcantarillado, ya que determina la escorrentía superficial que debe ser conducida tanto en volumen, caudal, tiempos para producir el pico.

Para mejorar el alcance de investigaciones futuras se recomienda mejorar la recopilación de datos primarios de la cuenca de estudio. Estos estudios se consideran de gran importancia en cuencas con altas pendientes y combinación de uso de suelos, dado que como se muestra en los resultados ambos factores afectan al tiempo de repuesta hidrológica de una cuenca, atenuando o acelerando esta.

\section{REFERENCIAS}

[1] E. Sardi, "Cambios socio-demográficos en Colombia: período intercensal 1993-2005," Revista de la información básica, vol. 4, pp. 13-27, Dic, 2007. 
[2] L. Leopold, "Hydrology for- Urban Land Planning A Guidebook on the Hydrologic," Geological Survey Circular, vol. 55, pp. 18, 1968.

[3] D. G. Anderson, "Effects of urban development on floods in northern Virginia," Geological Survey WaterSupply Paper, pp. 22, 1968.

[4] L. A. Martens, "Flood inundation and effects of urbanization in Metropolitan Charlotte North Carolina," Geological Survey Water-Supply Paper, p. 60, 1968.

[5] K. V Wilson, "Flood frequency of streams in Jackson, Mississippi," US Geological Survey, Rep 66155, 1966.

[6] R. W. Carter, "Magnitude and frequency of floods in suburban areas," Geological Survey Professional Paper, vol. 424-B, p. 9-11, 1961.

[7] S. W. Wiitala, "Some aspects of the effect of urban and suburban development upon runoff," US Geological Survey, Reston, VA, Rep 61-172, 1961.

[8] W. H. Espey, C. W. Morgan, and F. D. Masch, "Study of some effects of urbanization on storm runoff from a small watershed," Texas Water Development Board, Austin, TX, Rep 23, 1966.

[9] L. G. James y C. L. Larson, "Modeling infiltration and redistribution of soil water during intermittent application," Transactions of the ASAE, vol. 19, pp. 482-488, 1976.

[10]J. I. Vélez Upegui, "Desarrollo de un modelo hidrológico conceptual y distribuido orientado a la simulación de las crecidas," Tesis Doctoral, Escuela Técnica Superior de Ingenieros de Caminos, Canales y Puertos, Universidad Politécnica de Valencia, Valencia, 2001.

[11]M. Gómez Valentín, Curso de Hidrología Urbana, 1er ed. Barcelona, España: Distribuidora Alfambra de Papelería S.L., 2006.

[12]J. A. G. Jaimes y R. G. W. Venneker, "Simulación de los procesos de escorrentía en la cuenca de montaña de Terzieterbeek, Limburg - Holanda," Rev. UIS Ing., vol. 2, no. 2, pp. 105-112, 2003.

[13]J. G. Jaimes, S. G. Isidro, E. Niño, y Á. A. A. Archila, "Modelos hidrológicos en la cuenca experimental de Rio Sucio, municipio de Tona, Santander," Rev. UIS Ing., vol. 7, no. 1, pp. 23-29, 2008.
[14]W. H. Green y G. A. Ampt, "Studies on soil physics: 1. the flow of air and water through soils," The Journal of Agricultural Science, vol. 4, no. 1, pp. 1-24, 1911.

[15]V. Te Chow, D. R. Maidment, y L. W. Mays, Hidrología aplicada, Bogotá, Colombia: McGraw-Hill Interamericana SA Colombia, 1994.

[16]E. J. Sánchez Trujillo y J. Zambrano Nájera, "Cálculo de la infiltración mediante el método del número de curva modificado para cuencas urbanas de alta montaña, caso cuenca experimental San Luis-Manizales, Colombia, 4th IWA Mexico Young Water Profesional Conference 2015, Guanajuato, México, 2017, p. 849.

[17]M. Gómez Valentín, Curso de Análisis y rehabilitación de redes de alcantarillado mediante el código SWMM 5.0. Barcelona, España: Distribuidora Alfambra de Papelería S.L., 2007.

[18]IDEA, "Cuencas experimentales urbanas en Colombia caso Manizales. Fase 1. Caracterización e instrumentación,” Manizales, Colombia, 2005.

[19]IDEA, "Cuencas experimentales urbanas en Colombia caso Manizales. Fase 2. Complementación, Análisis e Interpretación de la Información," Dirección de Investigaciones Manizales DIMA, Manizales, Colombia, 2007.

[20]CIMOC y CEDERI, "Microzonificación Sísmica de la Ciudad de Manizales," Alcaldía de Manizales, Bogotá, Colombia, 2002.

[21]J. N. Suárez, "Propuesta metodológica para el estudio del proceso lluvia escorrentía en cuencas urbanas de ciudades de media montaña andina. Caso de estudio: cuenca experimental quebrada San Luís, Manizales Caldas," Tesis de Maestría, Departamento de Ingeniería Civil, Universidad Nacional de Colombia, Manizales, Colombia, 2008.

[22]W. J. Rawls y D. L. Brakensiek, "Estimation of Soil Water Retention and Hydraulic Properties," in Unsaturated Flow in Hydrologic Modeling: Theory and Practice: Springer Netherlands, 1989, cap. 1, vol. 3, pp. 275-300.

[23]USDA-ARS, Sitio Web STEWARDS (Sustaining the Earth's Watersheds - Agricultural Research Database System), [Online]. Available: http://www.nrrig.mwa.ars.usda.gov/stewards/stewards.h tml. 2008 
Estudio de la respuesta hidrológica en la cuenca urbana de montaña San Luis-Palogrande

[24]J. J. Vélez Upegui y A. Botero Gutiérrez, "Estimación del tiempo de concentración y tiempo de rezago en la cuenca experimental urbana de la quebrada San Luis, Manizales," Dyna, vol. 165, no. 165, pp. 59, Dec, 2011. 